\title{
Oral and Oropharyngeal HPV prevalence in South Africa \\ A systematic review and meta-analysis
}

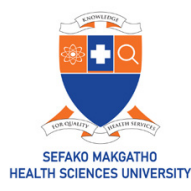

SADJ November 2021, Vol. 76 No. 10 p632 - p639

NH Wood', PD Motloba ${ }^{2}$, LN Makwakwa ${ }^{3}$, JP Bogers ${ }^{4}$

\begin{abstract}
Background

Prevalence data for HPV infection in the head and neck in Southern African populations is lacking. In addition to cervical cancer, this sexually transmitted oncogenic virus is responsible for a subset of head and neck cancer and is transmitted via oral sexual routes, and through other forms of intimate contact between anatomical sites lined by mucosa. This systematic review and meta-analyses aimed to synthesize data for the prevalence of head and neck HPV infection in South Africa. Original research papers from South Africa reporting on the prevalence of HPV in the head and neck was systematically reviewed using PubMed, Ovid Medline, Embase and the Cochrane Library. A meta-analysis on the prevalence data was conducted for 16 papers that met the inclusion criteria.
\end{abstract}

\section{Main text}

This systematic review and meta-analysis reports a pooled prevalence for head and neck HPV infection of $11 \%$. The study shows both a shortage of, and a data lag for HPV prevalence studies in the head and neck of Southern African populations. Technological improvement over time, differences in data collection methodology, differences in laboratory analysis processes and

\section{Author affiliations:}

1. Neil Hamilton Wood: Department of Periodontology and Oral Medicine, School of Oral Health Sciences, Sefako Makgatho Health Sciences University, Pretoria, South Africa, Neil.wood@smu.ac.za ORCID: 0000-0001-8950-7999

2. Pagollang David Motloba: Department of Community Dentistry, School of Oral Health Sciences, Sefako Makgatho Health Sciences University, Pretoria, South Africa, Pagollang.Motloba@smu.ac.za ORCID: 0000-0003-1379-7576

3. Louisa Nokukhanya Makwakwa: Clinical Manager, Lecturer, School of Oral Health Sciences, Sefako Makgatho Health Sciences University, Pretoria, South Africa, Nokukhanya.Makwakwa@smu. ac.za

4. John-Paul Bogers: Faculty of Medicine and Health Sciences, Applied Molecular Biology Research Group (AMBIOR), Laboratory of Cell Biology and Histology, University of Antwerp, Belgium. jpbogers@ambior.org; ORCID: 0000-0001-9107-132X

Corresponding author: Neil H Wood

neil.wood@smu.ac.za, BOX D26, Medunsa, 0204, South Africa

\section{Author Contribution Statement:}

NHW and JB conceptualized the work. NHW PDM and NLM made substantial contributions to the design of the work. NHW, NLM and PDM contributed towards the acquisition, analysis and interpretation of data. NHW and PDM wrote the first draft. NHW, JB and PDM provided substantial intellectual content and input. NHW, PDM, NLM and JB revised the work critically for important intellectual content. NHW, PDM, NLM and JB gave final approval of the version submitted for publication. NHW, PDM, NLM and JB agree to be accountable for all aspects of the work in ensuring that questions related to the accuracy or integrity of any part of the work are appropriately investigated and resolved. differences in the selection of study populations including various population risk levels all influence the prevalence measurement outcome.

\section{CONCLUSION}

Prevalence reporting for head and neck HPV in South Africa is lagging with only a few reports in existence over a 25-year period. The overall pooled prevalence of $11 \%$ is slightly higher than global averages. Populations comprising higher risk groups exhibit higher HPV prevalence rates which is in part influenced by the existence of comorbidities in these groups. Methodologic and study design consistencies and standardization will improve prevalence reporting in this geographic region.

Key Words: HPV prevalence, oropharynx, oral, HPV, human papillomavirus

\section{INTRODUCTION}

The Human papillomavirus (HPV) is classified as oncogenic to humans (group 1 infectious agent) and the oropharynx is an anatomic site associated with high-risk (HR)-HPV-induced epithelial transformation. ${ }^{1}$ This is the most common sexually-transmitted pathogen that includes oro-genital and oral-oral transmission routes. ${ }^{2}$ The virus infects exposed basal keratinocytes of a micro-lacerated or abraded mucosal surface. ${ }^{3}$

Clinically productive HPV infection of the skin or mucosae result in benign or malignant lesions. The benign HPV-induced lesions are typically single exophytic proliferations often described as having a cobblestone-like surface or finger-like projections. These lesions are usually of a normal mucosal coloring unless traumatized or secondarily infected. ${ }^{3}$ There are also reports that describe HPV association with oral leukoplakic lesions that exhibit dysplastic change, with oral lichen planus and with erythroplakia, although these may represent incidental findings. Associations with malignancies have been reported for cases of verrucous carcinomas. ${ }^{4-6}$ However, a subset of head-and-neck carcinomas driven by HPV infection as seen in younger, sexually active individuals is now well recognized. ${ }^{7-10}$

It appears that some HPV types exhibit stronger gender bias than others ${ }^{11-13}$, although this association may be artefactual or biased, as many HPV-prevalence studies focus purely on the cervix. In a study from the US, men had three times higher asymptomatic 


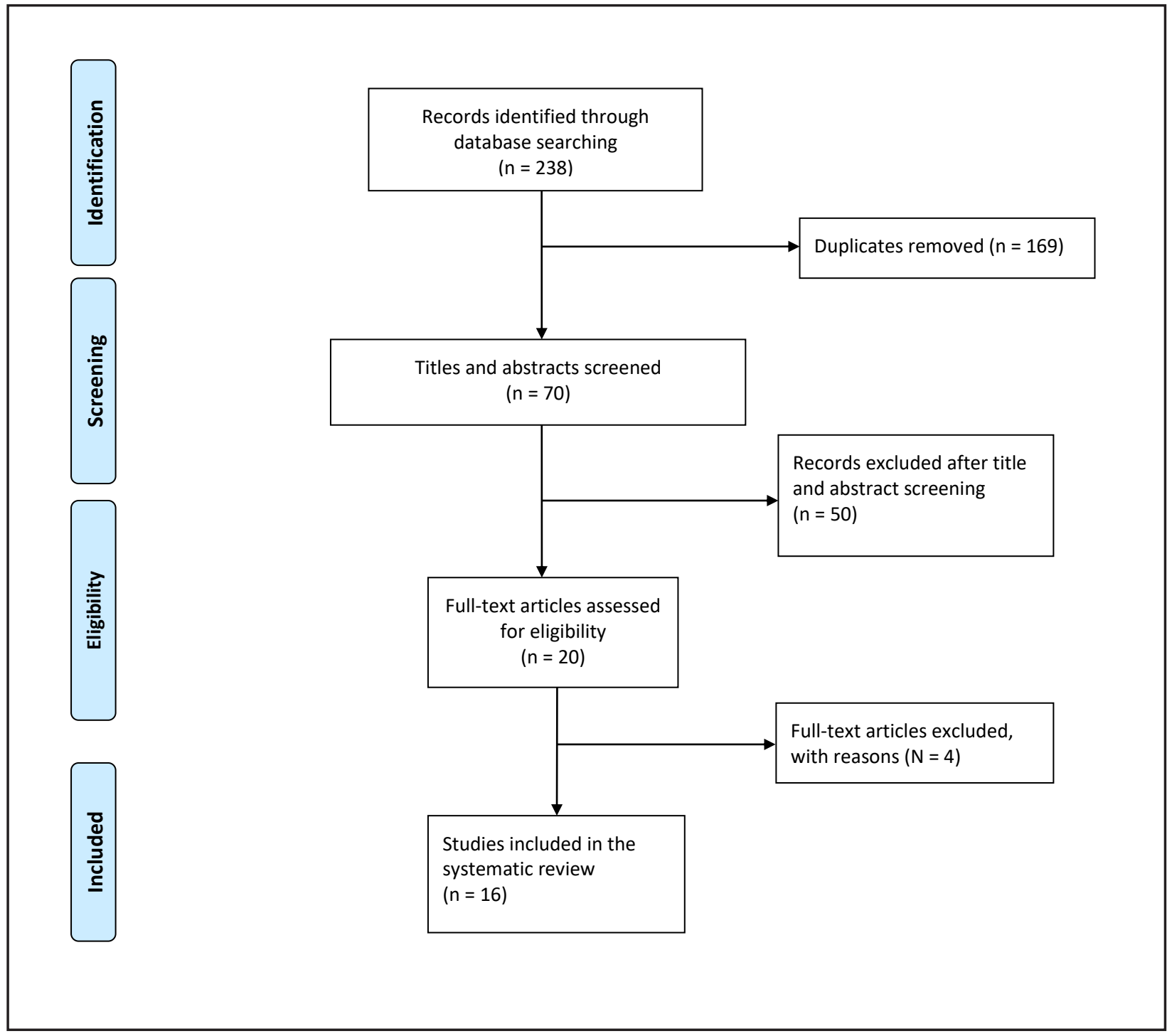

HPV infection than women. ${ }^{14}$ The reasons for this gender disparity remain unclear. $^{9}$

High-risk HPV-types may be found in benign HPV-associated oral lesions and low-risk (LR)-HPV types may be identified in malignant HPV-associated lesions like oropharyngeal SCC (OPSCC). ${ }^{15}$ Some view the presence of HPV in the mouth as being an oral HPV carrier, or as a passenger infection ${ }^{16-18}$; and these individuals are probable transmitters of the virus $^{19}$ through oral sex practices and through open mouth kissing among others. ${ }^{2}$

The prevalence of HPV in the mouth and oropharynx has been studied globally, but there is an apparent lack of substantial data from sub-Saharan Africa, and specifically, South Africa. A 2012 systematic review and meta-analysis that reported findings on malignancies resultant from infectious agents also found a significant lack of prevalence studies from developed areas. ${ }^{20}$ Only 3 South African studies were included in a 2018 systematic review ${ }^{9}$, and the Human papillomavirus and related diseases report cited only two South African studies, both dealing with oral/oropharyngeal squamous cell carcinoma. ${ }^{2}$
Given the epidemiologic shift in HR-HPV infection of the head and neck and its closer association with OPSCC $^{9}$, it becomes even more urgent to estimate the burden of HPV infection of South Africans in terms of incidence and persistence. The systematic synthesis of these epidemiologic data would improve our understanding of the diseases and of its natural course in this geographic setting.

\section{METHODOLOGY}

Search strategy

The Preferred Reporting Items for Systematic Reviews and Meta-analysis (PRISMA) was adopted in conducting this meta-analysis. The CoCoPop (Condition, Context and Population) framework was adopted to generate the following keywords Condition - Oral HPV, oropharyngeal HPV; Context - South Africa, English language; Population - Human subjects. Two authors (NHW and LNM) performed searches of PubMed, EBSCOhost, MEDLINE and Embase. For additional articles, grey literature, conference proceedings and reference lists from previously published research were reviewed. To augment the search, MeSH and Emtree subject headings were used individually and in combination. 


\begin{tabular}{|c|c|c|c|c|c|c|}
\hline Study & Date & Site & Population / Sample type & $\mathrm{N}$ & $\%$ Prevalence $(n)$ & Specimen \\
\hline Mbulawa et al. ${ }^{22}$ & 2014 & Oral & Heterosexual couples & 442 & $8,4(37)$ & Oral brush \\
\hline Boy et al. ${ }^{16}$ & 2006 & Oral and OP & Tissue & 59 & $11,9(7)$ & FFPE tissue blocks \\
\hline Chikandiwa et al. ${ }^{23}$ & 2018 & Oral and $\mathrm{OP}$ & $\mathrm{HIV}+\mathrm{Men}$ & 181 & $1,7(3)$ & Rinse and Gargle \\
\hline Muller et al. ${ }^{24}$ & 2016 & $\mathrm{OP}$ & MSM & 200 & $11,5(23)$ & OP brush \\
\hline Davidson et al. ${ }^{25}$ & 2014 & Oral and OP & Men & 125 & $5,6(7)$ & Rinse and Gargle \\
\hline Richter et al. ${ }^{26}$ & 2008 & Oral & HIV+ Women & 30 & $20(6)$ & Oral brush \\
\hline Vogt et al. ${ }^{27}$ & 2013 & Oral and OP & Heterosexual couples & 68 & $14,7(10)$ & Oral brush \\
\hline Paquette et al. ${ }^{18}$ & 2013 & $\mathrm{OP}$ & Tissue & 51 & $94,1(48)$ & FFPE tissue blocks \\
\hline Van Rensburg et al. ${ }^{28}$ & 1995 & Oral & Tissue & 66 & $1,5(1)$ & FFPE tissue blocks \\
\hline Van Rensburg et al..$^{29}$ & 1996 & Oral & Tissue & 146 & $3,1(2)$ & FFPE tissue blocks \\
\hline Mistry et al. ${ }^{30}$ & 2019 & Oral and OP & MSM & 199 & $6(12)$ & Rinse and Gargle \\
\hline Marais et al..$^{31}$ & 2008 & Oral & Women with cervical disease & 105 & $26,7(28)$ & Oral Brush \\
\hline Marais et al. ${ }^{32}$ & 2006 & Oral & Dental clinic attendees & 116 & $3,4(4)$ & Oral Brush \\
\hline Sekee et al. ${ }^{33}$ & 2018 & $\mathrm{OP}$ & Tissue & 20 & $5(1)$ & FFPE tissue blocks \\
\hline Bulane et al..$^{34}$ & 2020 & $\mathrm{OP}$ & Tissue & 449 & 7,3 (33) & FFPE tissue blocks \\
\hline Wood et al. ${ }^{2}$ & 2020 & $\mathrm{OP}$ & $\begin{array}{c}\text { Dental clinic attendees \& HIV+ } \\
\text { patients }\end{array}$ & 221 & $3,6(8)$ & Rinse and Gargle \\
\hline
\end{tabular}

\section{Study selection}

Studies were included if they satisfied the following criteria: (i) human subjects, (ii) described the incidence, prevalence of oral or oropharyngeal HPV (ii) South African population. The following studies were excluded (i) systematic reviews, (ii) meta-analyses, (iii) case control studies, (iv) case studies, (v) studies of nonhuman subjects.

\section{Quality Assessment}

The study quality was assessed using the Critical Appraisal Checklist for Studies Reporting Prevalence Data published by the JBI. The tool scores the studies on a total score of 9 points, with higher scores indicative of better design and quality. Two researchers (NHW and LNM) blindly performed assessed the studies and assigned a rating of "poor" $(\leq 3)$, "fair" (4-7), or "good" $(\geq 8)$ to each study. Only studies achieving a rating of good by both reviewers were included in the analysis. Any disagreement was resolved through discussion between the researchers, or by third reviewer (DPM).

\section{Data Extraction}

A form specially developed for this study was used by two independent authors to extract information. The variables of interest included: authors and date of publication; characteristics of the study population (gender, age, sexual orientation); site of sample collection (oral cavity, oropharynx), method of sample collection (oral brush, rinse and gargle, and tissues blocks), prevalence of HPV (crude prevalence and population size).

\section{Data analysis}

The measure of effect size (Prevalence of HPV) was computed using the Metaprop command for the meta-analysis of proportions in Stata ${ }^{\circledR}$. Metaprop is appropriate for proportions, which range from 0 or $100 \%$ and guarantees that Cls remain within the 0 to 1 range. This stability is achieved by using the binomial distribution to model within-study variability. In this study, the effect size was calculated together with the corresponding 95\% Cl using the Wald method executed with the cimethod (score) command. Forest plot was generated to show the individual and pooled effects size, 95\% Cl, the author's name, publication year and study weights (both for primary studies and this systematic review/meta-analysis), based on subgroups. The random effects model was used to compute the overall estimate of prevalence and the 95\% confidence interval. The Cochran's $\mathrm{I}^{2}$ index was calculated to measure heterogeneity among studies, with $p<0.05$ indicative of heterogeneity. The $\mathrm{I}^{2}$ values of $<25 \%$, between ( $25 \%$ and $50 \%$ ), and of $>50 \%$ reflected, mild, moderate and high heterogeneity respectively.

Subgroup analysis was performed to assess sources of heterogeneity among the studies. Variables included in the subgroup analysis included publication date, site and method of sample collection. Additional sensitivity analysis was done using the "leave-one-out" approach to evaluate the robustness of the pooled results. By removing one study at a time, the weighted or disproportional influence of a single study on the overall prevalence was evaluated. Publication bias was checked by visual inspection of funnel plots of prevalence and precision and statistical tests.

\section{RESULTS}

A total of 239 records were obtained from a comprehensive literature search. After the screening of 70 abstracts and titles, 20 full texts were reviewed, of which 16 satisfied the inclusion and exclusion criteria and were included in the analysis (Figure 1). The 16 studies were published between 1995 and 2020 with the total sample size of 2478 and 230 confirmed cases of HPV (Table 1). Larger heterogeneity was anticipated for the small South African data pool.

Heterogeneity assessment

Heterogeneity of the studies was assessed using Cochran's $Q$ and $I^{2}$ statistics. The random-effects model was adopted to pool the study-specific prevalence rates 
Table 2: Subgroup analysis of oral HPV by site, specimen, and date

\begin{tabular}{|c|c|c|c|c|}
\hline Subgroup & (n) studies & Prevalence (\%) & 95\% Confidence Interval & $\begin{array}{c}\text { Heterogeneity } \\
\mathrm{I}^{2}(\%) ; \quad \mathrm{p} \text {-value }\end{array}$ \\
\hline Overall & 16 & 11.0 & $6.0-17.0$ & $95.07 ; 0.000$ \\
\hline \multicolumn{5}{|l|}{ Site } \\
\hline Oral & 6 & 8.0 & $2.0-16.0$ & $91.03 ; 0.00$ \\
\hline Oropharynx & 4 & 27.0 & $3.0-62.0$ & $98.49 ; 0.00$ \\
\hline Oral \& Oropharynx & 6 & 6.0 & $3.0-10.0$ & $74.50 ; 0.00$ \\
\hline \multicolumn{5}{|l|}{ Specimen type } \\
\hline Rinse \& Gargle & 4 & 4.0 & $2.0-7.0$ & $49.21 ; 0.12$ \\
\hline Oral Brush & 6 & 13.0 & $7.0-19.0$ & $85.31 ; 0.00$ \\
\hline FFPE tissue blocks & 6 & 16.0 & $1.0-40.0$ & $97.87 ; 0.000$ \\
\hline \multicolumn{5}{|l|}{ Date of publication } \\
\hline Before 2010 & 6 & 8.0 & $2.0-19.0$ & $91.21 ; 0.000$ \\
\hline 2010 and beyond & 10 & 12.0 & $5.0-22.0$ & $96.35 ; 0.000$ \\
\hline \multicolumn{5}{|l|}{ Sensitivity analysis } \\
\hline$n-1^{18}$ & 15 & 7.0 & $4.0-10.0$ & $85.57 ; 0.000$ \\
\hline$n-2^{18,31}$ & 14 & 7.5 & $5.1-11.6$ & $72.13 ; 0.000$ \\
\hline
\end{tabular}

and adjust for variability, attributable to large heterogeneity $\left(P<0.000\right.$ and $\left.I^{2}=95.07\right)$. To better understand the methodological and clinical variation we undertook subgroup according to source and type of specimen type, publication date and risk profile.

\section{Subgroup and sensitivity analysis}

Prevalence statistics for the pooled HPV studies $(n=16)$, as well subgroup and sensitivity analysis estimates are summarized in table 2. The estimated overall prevalence of the HPV was 0.11 (95\% Cl = 0.06 - 0.17). The sensitivity analysis was furthered by removing two studies ${ }^{18,31}$, which reported significantly higher prevalence and small sample sizes (table 2). Subgroup analyses revealed that HPV was more prevalent in specimens that were sourced from the oropharynx $(0.237 ; 95 \% \mathrm{Cl}=0.059-0.67)$ than in those from the oral cavity $(0.072 ; 95 \% \mathrm{Cl}=0.03-$ $0.163)$, and in the case where specimens were sourced from both sites $(0.069 ; 95 \% \mathrm{Cl}=0.037-0.125)$. Similarly, studies that were published before 2010, reported higher prevalence than the ones published in the last 10 years, $(0.237 ; 95 \% \mathrm{Cl}=0.059-0.67)$ and $(0.237 ; 95 \% \mathrm{Cl}=0.059-0.67)$ respectively. Equally, the prevalence differed according to the level of risk and type of specimen used in the detection of HPV. These proportions did not differ significantly among the subgroups except for specimen type $(p=0.044)$ (Table 2).

The exclusion of the outlier study by Paquette and colleagues $^{18}$, resulted in significant decrease in prevalence, $(0.07 ; 95 \% \mathrm{Cl}=0.04-0.1)$ (Table 2). The additional exclusion of study by Marais et al. ${ }^{31}$ had a negligible influence of the HPV prevalence rate, $(0.078 ; 95 \% \mathrm{Cl}=0.051-0.118)$. Overall, these two studies resulted in a $33.3 \%$ increase in the overall estimate of HPV $(7.8 \%$ to $10.4 \%)$, which provides critical explanation on the source of heterogeneity.

\section{DISCUSSION}

The prevalence of HPV infection of the head and neck is well documented globally. However, these data are not representative of the South African community. Among the reasons for this is the relative lack of analyses from general populations in South Africa, with the bulk of the studies reporting on HPV detection in the head and neck coming from focussed groups, all with varying confounding factors that contribute towards HPV acquisition (Table 1). Several meta-analyses have been done over several years with very few to no papers being included from the South African setting. The 2010 systematic review by Kreimer and colleagues included only one paper from South Africa. ${ }^{35}$ The Human Papillomavirus and Related Diseases Report cited 2 South African studies, but these were not included in the 9 studies found suitable to present a picture of the global HPV burden in the head and neck. ${ }^{21}$

The detection of HPV-DNA does not imply infection by HPV but may represent a passenger infection or carrier status. Some individuals may however harbour asymptomatic, but definite HPV infection of which a smaller portion, usually HR-HPV persists, and subsequently increases the risk for malignant transformation. ${ }^{3,18}$ Persistence data for HR-HPV infection of the head in neck in South Africans are lacking.

Specimen collection, sampling, analyses methods Patient demographics, geographic location, population risk profiles, sampling methods, anatomical sites sampled, tissue specimen type and quality, storage and transport, and detection methods all influence the reported prevalence rates of HPV-infection. ${ }^{3,9,13,26,36}$ The salivary detection of HPV is influenced by immune efficiency, smoking and the sensitivity and specificity of the PCR-technique applied. ${ }^{19,37}$ Detection frequency of different HPV types is also influenced by the specific intra-oral site infected, and the presence or absence of oral diseases such as periodontitis or oral lichen planus that expose basal keratinocytes. ${ }^{3,38}$ Oral wash specimens do not allow for oral site discrimination but come into contact with portions of the oropharynx and can therefore not be considered as an exclusively oral specimen. 


\section{Prevalence of HPV in South Africa}

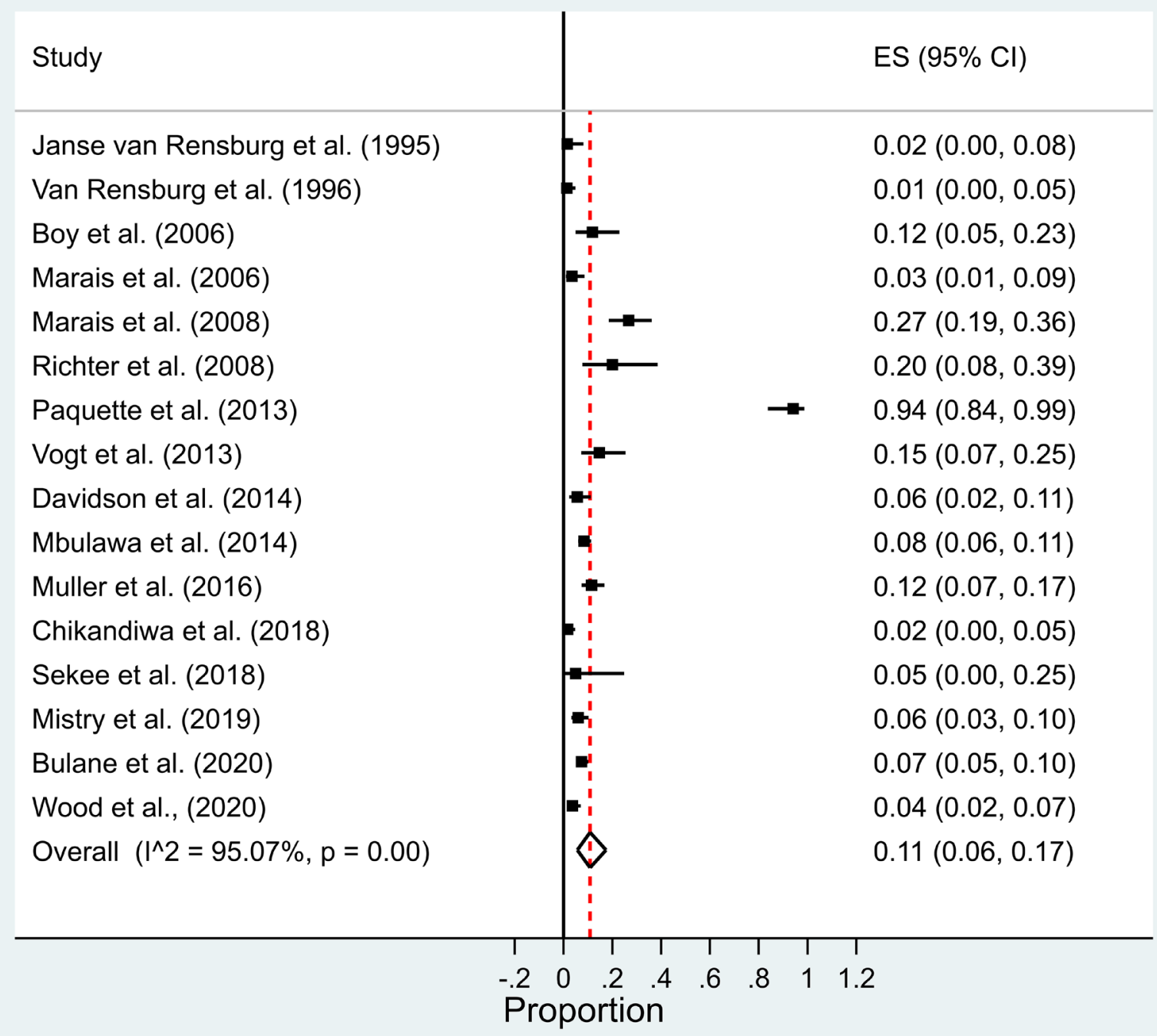

\section{Publication Bias}

The risk of publication bias was studied by funnel plot analysis, Egger's test and Begg's correlation. The statistical tests point to absence of bias; Egger's test $(p=0.415)$, Begg's $(p=0.293)$ respectively. We conclude that there is no evidence of publication bias in this meta-analysis. Therefore, the large heterogeneity cannot be attributed to publication bias, but rather to clinical and methodological variations among the included studies.

This meta-analysis validates the impact of various study designs and conduct on the estimation of the prevalence of HPV. The observed heterogeneity can be reasonably attributed to differences in clinical and methodological differences, and to some extent population risk level. The heterogeneity of the published papers makes a meaningful systematic review highly challenging, yet necessary. ${ }^{13}$

Data on HPV-associated malignancies of the head and neck:

HPV-16 carcinogenicity is established for oral/ oropharyngeal and laryngeal squamous cell carcinoma (SCC) and is considered a requisite aetiologic factor for a molecular and clinically distinct subset of head and neck SCC. . $20,21,35$ Proportionally, oral/oropharyngeal cancers that originate from HR-HPV infection may be small, but HPV-16 is responsible for the vast majority of these SCC (40-60\%). ${ }^{35,39}$ Data from the U.S. clearly shows that HPV-driven head and neck SCC has overtaken cervical SCC. ${ }^{9}$

Data on oral and oropharyngeal cancer from Africa is limited when compared to contributions from other geographic regions, and is usually presented as small series. ${ }^{27,40}$ A systematic review published in 2013 on prevalence of HPV-infection in oropharyngeal and non-oropharyngeal head and neck malignancies reported no data from Africa and state that the research on HPV-infection in these lesions are needed from Africa. ${ }^{13}$ Similarly, the meta-analyses by Dayyani and colleagues ${ }^{41}$ and by de Martel and co-authors ${ }^{20}$ did not include any data from Southern Africa. The Human papillomavirus and related diseases report ${ }^{21}$ cited only two papers ${ }^{16,29}$ from South Africa with regard to HPV-associated head and neck SCC further highlighting the lack of reliable data from South Africa. 
Evaluation of case-control studies in which tumour tissue was analysed for HPV E6 and E7 oncoprotein expression showed that the HR-HPV was prevalent in $13 \%$ of lesion tissue in studies originating outside of North America, Europe, Australia, and Japan. ${ }^{20}$ The South African studies reporting HPV detection in oral/oropharyngeal SCC tissue specimens reflect an unfeasibly wide range of $1.4 \%$ to $94.1 \%$ (Table 1 ). Due to this high level of heterogeneity, subgroup analysis could not be undertaken to estimate the pooled prevalence of HPV related to the SCC. The prevalence of oral and oropharyngeal mucosal HPV infection remains undescribed for the general South African population, despite the existence of smaller sampled cohorts.

\section{Systematic review}

The association between HPV and oral and or pharyngeal carcinoma necessitates the estimation of HPV presence in a variety of population subgroups. Large heterogeneity renders the computation of the summary estimate challenging. The lack of South African data is demonstrated by the effort to consolidate a detailed systematic review from the diverse assortment of study types in the limited pool of contributing studies. The clinical and methodological heterogeneity between the studies included in this systematic review varied greatly. First, the lag bias which is characterised by delayed publication of HPV papers in South Africa extends over 15-year period. This phenomenon could account for differences in diagnostic and identification of HPV as underpinned by advanced in technology and developed protocols. The prevalence of HPV increased from an average of $8.0 \%$ in the 1990 s to $12.0 \%$ a decade later. Six studies compared to ten were published in these respective periods, suggesting the presence of publication bias. Similar findings in the systematic review of 66 papers by Tam and colleagues $^{9}$, indicated the increase in HPV prevalence from $3.0 \%$ to $7.9 \%$ in the 1990's to the 2010's. Secondly, variations in methodologies, population risk profiles and geographic location differences, choice of anatomical sites and specimens, and differences in laboratory investigations may reflect the passage of time, thus further accounting for greater heterogeneity. Despite the design shortcomings, this meta-analysis represents the recent attempt to provide a summarised estimate of the burden of the prevalence of oral and oropharyngeal HPV in South Africa.

The prevalence of HPV detection ranges between $6.2 \%$ and $17.7 \%$ compared to our estimate of $11.0 \% .42,43$ However, detection increases when studying groups with associated risk factors for HPV transmission/ infection. An HPV prevalence of $10 \%$ and more has been reported in studies among high-risk men who have sex with other men and also in women diagnosed with cervical diseases. In HIV-seropositive patients, head and neck HPV-detection can be up to $30 \%$, with chances for oral detection of HPV being up to three times higher in HIV-seropositive patients. ${ }^{44,45}$ Benign and potentially malignant oral lesions are associated with HPV prevalence estimates of around 18.6\% and $24.5 \%$ respectively. ${ }^{46}$ Trzcinska and colleagues reported $29 \%$ of head and neck squamous papillomas as positive for HPV. ${ }^{47}$ The higher approximation of HPV in our study could therefore be attributed to the inclusion of populations at high risk, the deployment of more sensitive diagnostic techniques in the latter decade and increasing the spectrum of any HPV subtype identification. As reported in table 1, the effects of specimen collection and processing, had a major impact on the high HPV estimates and observable heterogeneity.

\section{CONCLUSION}

The pooled estimate of $11 \%$ from the limited number of South African studies provides a reasonable approximation of HPV prevalence considering the limitations shown in this study. However, when removing one outlier study with considerable weighting, the prevalence rate is significantly reduced to $7 \%$. The increasing prevalence of head and neck HPV among the high-risk groups is attributable to coexistence with comorbidities. The improvement in technology over time has increased the positive detection rate, which could explain the spike in the reported prevalence. This meta-analysis demonstrated the effect of knowledge lag on the estimation of HPV prevalence. To counteract this phenomenon, studies must be standardised and must use the most robust protocols for the detection of HPV. This will permit comparability and computation of reliable estimates of prevalence for the South African population.

Prospective incidence and persistence studies are critical for other vulnerable or high-risk population groups to determine whether preventive vaccination is required, or exclusive vaccination of the female population would confer sufficient protection in these particular groups.

\section{Declarations}

Competing interests - Authors confirm that there are no competing interests to declare

\section{Acknowledgements}

This work was supported by the Sefako Makgatho Health Sciences University and the Flemish government (IUC-VLIR-OUS). The Authors also acknowledge the support of the WAKA-HPV network.

\section{REFERENCES}

1. IARC. Monographs on the evaluation of carcinogenic risks to humans, volume 100. A review of carcinogen-Part B: biological agents. Lyon: International Agency for Research on Cancer, 2011.

2. Wood NH, Makua KS, Lebelo RL, Redzic N, Benoy I, Vanderveken OM, et al. Human papillomavirus prevalence in oral and oropharyngeal rinse and gargle specimens of dental patients and of an HIV-positive cohort from Pretoria, South Africa. Adv in Virol. 2020; ID 2395219; doi. org/10.1155/2020/2395219

3. Syrjänen S. Oral manifestations of human papillomavirus infections. Eur J Oral Sci. 2018; 126(S1):49-66.

4. Syrjänen KJ. Human papillomavirus (HPV) infections and their associations with squamous 
cell neoplasia. Arch Geschwulstforsch 1987; 57:417-444.

5. Syrjänen $S$, Lodi $G$, von Bültzingslöwen I, Aliko A, Arduino P, Campisi G, et al. Human papillomaviruses in oral carcinoma and oral potentially malignant disorders: a systematic review. Oral Dis 2011; 17(S1):58-72.

6. Grce M, Mravak-Stipetic M. Human papillomavirus-associated diseases. Clin Dermatol 2014; 32:253-258.

7. Chaturvedi AK, Anderson WF, Lortet-Tieulent $J$, Curado MP, Ferlay J, Franceschi S, et al. Worldwide trends in incidence rates for oral cavity and oropharyngeal cancers. J Clin Oncol 2013; 34:4550-4554.

8. Leemans CR, Snijders PJ, Brakenhoff $\mathrm{RH}$. The molecular landscape of head and neck cancer. Nature Rev 2018; 18:269-282.

9. Tam S, Fu S, Xu L, Krause KJ, Lairson DR, Miao H, et al. The epidemiology of oral human papillomavirus infection in healthy populations: A systematic review and meta-analysis. Oral Oncol 2018; 82:91-99.

10. Yete S, D'Souza W, Saranath D. High-risk Human papillomavirus in oral cancer: Clinical implications. Oncology 2018; 94:133-141.

11. Komloš KF, Kocjan BJ, Košorok $P$, Luzar $B$, Meglič L, Potočnik $M$, et al. Tumor-specific and gender-specific pre-vaccination distribution of human papillomavirus types 6 and 11 in anogenital warts and laryngeal papillomas: A study on 574 tissue specimens. J Med Virol 2012; 84:1233-1241.

12. Danielewski JA, Garland SM, McCloskey J, Hillman RJ, Tabrizi SN. Human papillomavirus type 6 and 11 genetic variants found in 71 oral and anogenital epithelial samples from Australia. PLOS ONE 2013; 8:e63892; doi.org/10.1371/journal.pone.0063892

13. Mehanna $H$, Beech $T$, Nicholson T, El-Hariry I, McConkey C, Paleri V, et al. Prevalence of Human papillomavirus in oropharyngeal and nonoropharyngeal head and neck cancer - systematic review and meta-analysis of trends by time and region. Head \& Neck 2013; 35:747-755.

14. Sanders AE, Slade GD, Patton LL. National prevalence of oral HPV infection and related risk factors in the U.S. adult population. Oral Dis 2012; 18:430-441.

15. Prabhat MP, Lakshmi CR, Madhavi NS, Bhavana SM, Sarat G, Ramamohan K. Multifocal epithelial hyperplasia of oral cavity expressing HPV 16 gene: a rare entity. Case Reports in Dent 2013; doi. org/10.1155/2013/871306

16. Boy S, Janse van Rensburg E, Engelbrecht S, Dreyer L, Van Heerden M, Van Heerden W. HPV detection in primary intra-oral squamous cell carcinomas - commensal, aetiological agent or contamination? J Oral Pathol Med 2006; 35:86-90.

17. Fatahzadeh M, Schlecht NF, Chen Z, Bottalico D, McKinney S, Ostoloza J, et al. Oral human papillomavirus detection in older adults who have human immunodeficiency virus infection. Oral Surg Oral Med Oral Pathol Oral Radiol 2013; 115:505-514.

18. Paquette C, Evans MF, Meer SS, Rajendran V, Adamson CS, Cooper K. Evidence that alpha-9 human papillomavirus infections are a major etiologic factor for oropharyngeal carcinoma in black South Africans. Head and Neck Pathol 2013; 7:361-372.

19. Gaester K, Fonseca LA, Luiz O, Assone T, Fontes AS, Costa $F$, et al. Human papillomavirus infection in oral fluids of HIV-1-positive men: prevalence and risk factors. Sci Rep 2014; 4:6592.

20. De Martel C, Ferlay J, Francheschi S, Vignat J, Bray F, Forman D, et al. Global burden of cancers attributable to infections in 2008: a review and synthetic analysis. Lancet Oncol 2012; 13:607-15.

21. Bruni L, Albero G, Serrano B, Mena M, Gómez $D$, Muñoz J, et al. ICO/IARC Information Centre on HPV and Cancer (HPV Information Centre). Human Papillomavirus and Related Diseases in the World. Summary Report, 17 June 2019. https:// www.hpvcentre.net/statistics/reports/XWX.pdf, (accessed 3 August 2020).

22. Mbulawa ZZA, Johnson LF, Marais DJ, Coetzee D, Williamson A. Risk factors for oral human papillomavirus in heterosexual couples in an African setting. J Infect 2014; 68:185-189.

23. Chikandiwa A, Pisa PT, Chersich MF, Muller EE, Mayaud P, Delaney-Moretlwe S. Oropharyngeal HPV infection: prevalence and sampling methods among HIV-infected men in South Africa. Int J STD \& AIDS 2018; 29:776-780.

24. Muller EE, Rebe K, Chirwa TF, Struthers H, Mclntyre $J$, Lewis DA. The prevalence of human papillomavirus infections and associated risk factors in men-who-have-sex-with-men in Cape Town, South Africa. BMC Infect Dis 2016; 16:440.

25. Davidson CL, Richter KL, van der Linde M, Coetsee J, Boy SC. Prevalence of oral and oropharyngeal human papillomavirus in a sample of South African men: A pilot study. SAMJ 2014; 104:358-361.

26. Richter $\mathrm{KL}$, Janse van Rensburg $\mathrm{E}$, van Heerden WFP, Boy SC. Human papilloma virus types in the oral and cervical mucosa of HIV-positive South African women prior to antiretroviral therapy. J Oral Path Med 2008; 37:555-559.

27. Vogt SL, Gravitt PE, Martinson NA, Hoffman J, D'Souza G. Concordant oral-genital HPV infection in South Africa couples: evidence for transmission. Frontier Oncol: Cancer Epidem Prev 2013; 3:1-7.

28. Van Rensburg EJ, van Heerden WF, Venter EH, Raubenheimer EJ. Detection of human papillomavirus DNA with in situ hybridisation in oral squamous carcinoma in a rural black population. S Afr Med J 1995; 85:894-896.

29. Van Rensburg EJ, Engelbrecht S, Van Heerden WF, Raubenheimer EJ, Schoub BD. Human papillomavirus DNA in oral squamous cell carcinomas from an African population sample. Anticancer Res 1996; 16:969-973.

30. Mistry HB. Dissertation: Prevalence of oral and oro-pharyngeal Human papillomavirus infection in a selected cohort of men from a rural/semi-rural area in Gauteng, South Africa. Sefako Makgatho Health Sciences University, Pretoria, South Africa, 2011. https://www.smu.ac.za/library/

31. Marais DJ, Passmore DS, Denny L, Sampson C, Allan BR, Williamson A. Cervical and oral Human papillomavirus types in HIV-1 positive and negative 
women with cervical disease in South Africa. J Med Virol 2008; 80:953-959.

32. Marais DJ, Sampson C, Jeftha A, Dhaya D, Passmore J, Denny $L$, et al. More men than women make mucosal IgA antibodies to Human papillomavirus type 16 (HPV-16) and HPV-18: a study of oral HPV and oral HPV antibodies in a normal healthy population. BMC Infect Dis 2006; 6:95.

33. Sekee TR, Burt FJ, Goedhals D, Goedhals J, Munsamy Y, Seedat RY. Human papillomavirus in head and neck squamous cell carcinomas in a South African cohort. Papillomavirus Res 2018; 6:58-62.

34. Bulane A, Goedhals D, Seedat RY, Goedhals J, Burt F. Human papillomavirus DNA in head and neck squamous cell carcinomas in the Free State, South Africa. J Med Virol 2020; 92:227-233.

35. Kreimer AR, Bhatia RK, Messeguer AL, González, P, Herrero, R, Giuliano, AR. Oral Human papillomavirus in healthy individuals: A systematic review of the literature. Sex Transm Dis 2010; 37:386-391.

36. Gillison ML, Broutian T, Pickard RK, Tong Z, Xiao W, Kahle L, et al. Prevalence of oral HPV infection in the United States, 2009-2010. JAMA 2012; 307:693-703.

37. Adamopoulou M, Viaraktaris E, Nkenke E, Avgoustidis D, Karakitsos P, Sioulas V, et al. Prevelance of human papillomavirus in saliva and cervix of sexually active women. Gynecol Oncol 2013; 129:395-400.

38. Pinheiro RS, de Franca TR, Rocha B, Ferreira DC, Ribeiro CMB, Cavalcanti SMB, et al. Human papillomavirus coinfection in the oral cavity of HIV-infected children. J Clin Pathol 2011; 64:1083-1087.

39. D'Souza G, Kreimer AR, Viscidi R, Pawlita M, Fakhry C, Koch WM, et al. Case-control study of human papillomavirus and oropharyngeal cancer. $\mathrm{N}$ Engl J Med 2007; 356:1944 -1956.

40. Warnakulasuriya S. Global epidemiology of oral and oropharyngeal cancer. Oral Oncol 2008; 45:309-316.

41. Dayyani F, Etzel CJ, Liu M, Ho C, Lippman SM, Tsao AS. Meta-analysis of the impact of human papillomavirus (HPV) on cancer risk and overall survival in head and neck squamous cell carcinomas (HNSCC). Head Neck Oncol 2010; 2:15.

42. Mravak-Stipetić M, Sabol I, Kranjčić J, Knežević M, Grce M. Human papillomavirus in the lesions of the oral mucosa according to topography. PLOS ONE 2013; 8:e69736.

43. Matos, LL, Miranda GA, Cernea CR. Prevalence of oral and oropharyngeal human papillomavirus infection in Brazilian population studies: a systematic review. Brazil J Otorhinolaryngol 2015; 81:554-567.

44. D'souza G, Carey TE, William WN Jr, Nguyen ML, Ko EC, Ridell J IV, et al. Epidemiology of head and neck squamous cell cancer among HIV-infected patients. J Acquir Immune Defic Syndr 2014; 65:603-610.

45. Beachler DC, D'Souza G. Oral HPV infection and head and neck cancers in HIV-infected individuals. Curr Opin Oncol 2013; 25;503-510.

46. Jayaprakash V, Reid M, Hatton E, Merzianu M, Rigual N, Marshall J, et al. Human papillomavirus types 16 and 18 in epithelial dysplasia of oral cavity and oropharynx: a meta-analysis, 1985-2010. Oral Oncol 2011; 47:1048-1054.

47. Trzcinska A, Zhang W, Gitman M, Westra WH. The Prevalence, Anatomic Distribution and Significance of HPV Genotypes in Head and Neck Squamous Papillomas as Detected by Real-Time PCR and Sanger Sequencing. Head and Neck Pathol 2020; 14:428-434 